architecture and microenviroment. In this study, we aim to generate multisite PDOs to demonstrate the spatial heterogeneity of cancer stem cells (CSCs) and responses to chemotherapy treatments in disseminated HGSOC.

Methodology Tumour cells were extracted from disseminated multisite tumours from chemo-naïve patients undergoing maximal effort cytoreductive surgery at Hammersmith Hospital, UK. PDOs were established by embedding tumour cells in basement membrane extract and in specialised organoid or RSpondin-enriched media. PDOs were propagated and treated with standard-of-care chemotherapy drugs (cis- and carboplatin, PARPi) to assess drug responses. PDOs were histologically processed for characterisation of tumour markers, and CSCs across multiple tumour sites and passages.

Result(s)* PDOs were established from multisite deposits ( $\mathrm{n}=8$ patients, mean $=7$ tumours per patient, range $=4-10)$ and confirmed to be of HGSOC tumour origin. Response to RSpondin-enriched media varied across sites and across patients, but was maintained over multiple passages. PDOs were assessed for expression of known CSC biomarkers (ALDH1, CD117, CD133, CD44). IC50 assays established for standardof-care chemotherapy drugs demonstrated heterogenous responses to treatment.

Conclusion* PDOs demonstrated the heterogeneity of the CSC population, growth conditions, and drug responses, reflecting the complexity of HGSOC. Our data suggests that treatment regimens chosen based on drug response from a single tumour site may not be effective against other disseminated tumours. PDOs which include multiple metastatic sites may lead to the development of targeted and personalized treatment strategies which reflect the spatial heterogeneity of HGSOC.

\section{MOLECULAR LANDSCAPE IN HIGH-GRADE SEROUS OVARIAN CANCER: THE CORRELATION BETWEEN BRCA MUTATIONAL STATUS AND STROMAL P16 EXPRESSION}

${ }^{1} \mathrm{M}$ Caretto*, ${ }^{2} \mathrm{~S}$ Pistolesi, ${ }^{2} \mathrm{AG}$ Naccarato, ${ }^{1} \mathrm{~T}$ Simoncini, ${ }^{1} \mathrm{~A}$ Gadducci. ${ }^{1}$ University of Pisa, Department of Clinical and Experimental Medicine, Division of Obstetrics and Gynecology, Pisa, Italy; ${ }^{2}$ University of Pisa, Department of Translational Research and New Technologies in Medicine and Surgery, Division of Surgical Pathology, Pisa, Italy

\subsection{6/ijgc-2021-ESG0.599}

Introduction/Background* The effect of BRCA mutational status on biological feature and survival outcome in advancedstage (IIB-IV stage) High-grade serous ovarian cancer (HGSOC) seems to confer a better prognosis. The aim of our preliminary work is to investigated the stromal p16 expression for HGSOC in advanced-stage (IIB-IV stage), using immunohistochemistry, and differences in the expression status depending on the mutational status of BRCA. Alterations in $\mathrm{p} 16$ protein expression have been reported to be associated with tumor development and progression. p16 expression status in the peritumoral stroma has been rarely investigated.

Methodology This is a retrospective study included 33 patients diagnosed with FIGO stage IIB-IV HGSOC who underwent primary debulking surgery (PDS) at the Departments of Gynecology and Obstetrics of the University of Pisa between January 2019 and April 2020. The stromal p16 expression in HGSOC was investigated by using immunohistochemistry, and the differences in p16 immunoreactivity linked with the BRCA mutational status were analyzed.

Result(s)* BRCA1/2 mutations were observed in 15 women (45\%). Wild-type -BRCA advanced HGSOCs exhibited diffuse, moderate-to-strong p16 immunoreactivity in $46.66 \%$ of cases, instead mutated-BRCA advanced HGSOCs showed elevated stromal p16 expression in $33.33 \%$ of cases.

Conclusion* This study introduces a new and interesting result: the correlation between stromal expression of p16 and BRCA mutational status. The majority of malignant lesions exhibited diffuse and moderate-to-strong p16 immunoreactivity, suggesting that stromal p16 expression can be used as an adjunctive predictive and prognostic biomarker for HGSOC. Further studies are necessary to confirm our preliminary results and to explain the different outcome of wt- and mBRCA HGSOC.

\section{THE ROLE OF DAPK1 IN THE CELL CYCLE REGULATION OF CERVICAL CANCER CELLS AND IN RESPONSE TO TOPOTECAN}

${ }^{1} \mathrm{~K}$ Gasimli*, 'M Raab, 'S Becker, 'M Sanhaji, 1,2K Strebhardt. 'Goethe University Frankfurt, Department of Gynecology and Gynecological Oncology, Frankfurt, Germany; ${ }^{2}$ German Cancer Consortium (DKTK), German Cancer Research Center, Partner Site Frankfurt am Main, Frankfurt, Germany

\subsection{6/ijgc-2021-ESG0.600}

Introduction/Background* Cervical cancer is the fourth most common cancers among women worldwide. Primary therapy of cervical cancer depends on the disease extent and is based on radical hysterectomy or chemoradiation. However, therapeutical approaches in metastatic and recurrent disease of cervical cancer are limited. Particular effort in drug development focuses on essential serine/threonine kinases like the death-associated protein kinase 1 (DAPK1) and Polo-like kinase 1 (PLK1) as potential therapeutic targets in cervical cancer.

Methodology and Result(s)* Our study examined the role of DAPK1 during the cell cycle of cervical cancer cells. We found that DAPK1 is autophosphorylated in mitosis, exhibiting only low activity towards exogenous substrates. Furthermore, DAPK1 localizes together with PLK1 at centrosomes, which can phosphorylate DAPK1. Finally, we could show that Topotecan, which is used in different clinical trials to treat cervical cancer, induces cell death, which partially depends on DAPK1. Conclusion* Topotecan is an effective drug for the treatment of cervical cancer. We explored the role of DAPK1 in Topotecan-induced cervical cancer cell death and revealed that the RNAi-based silencing of DAPK1 downregulates the apoptotic activity suggesting that DAPK1 could be a biomarker for the response to Topotecan in clinical trials. 
A

DAPK1 level

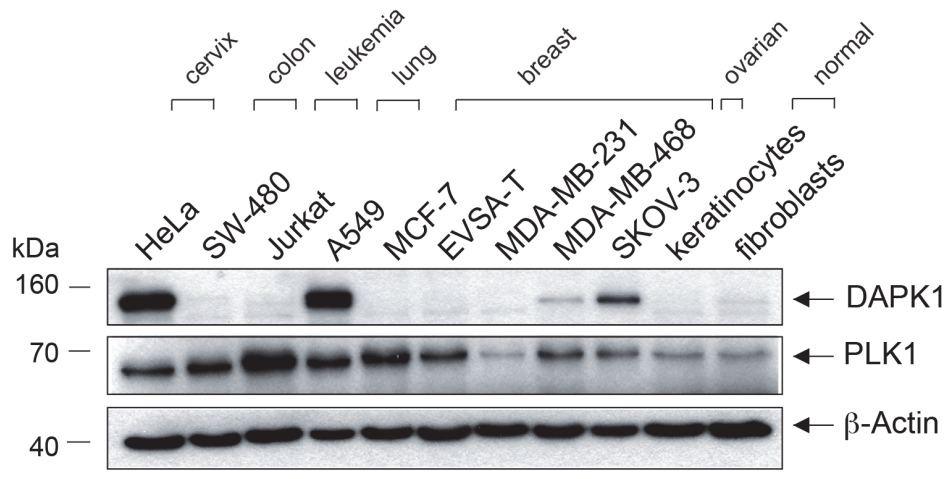

B

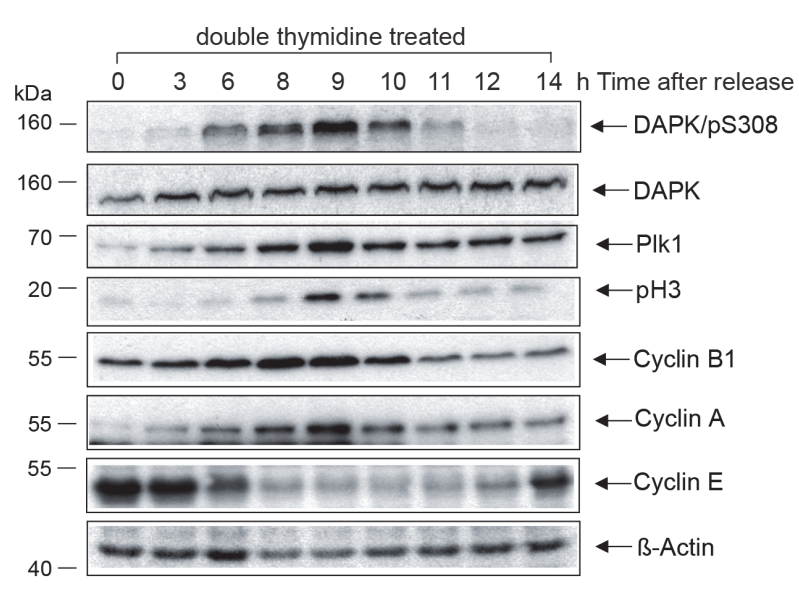

D

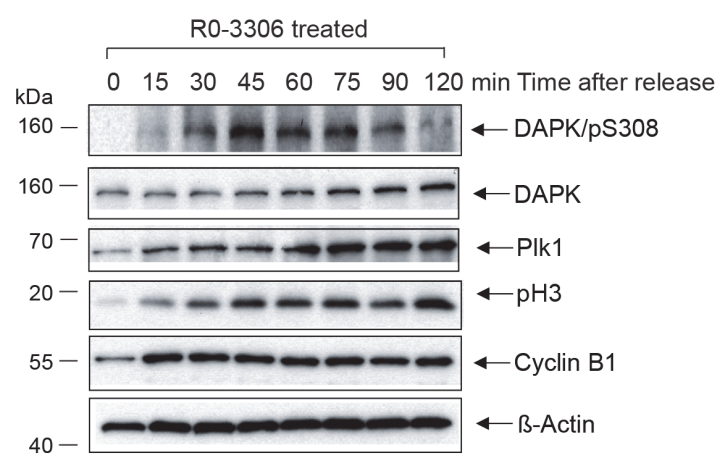

C

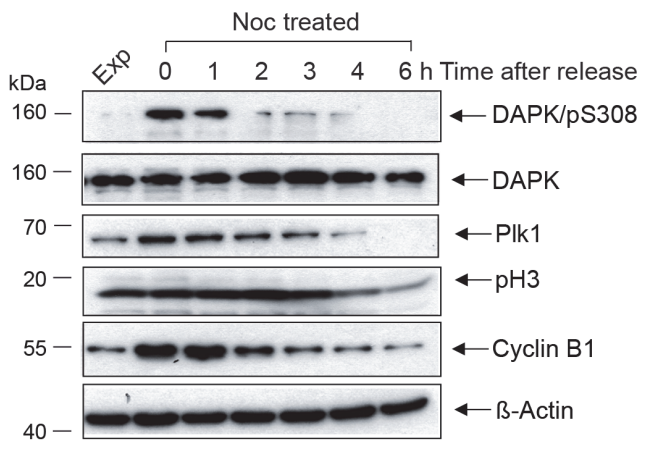

E

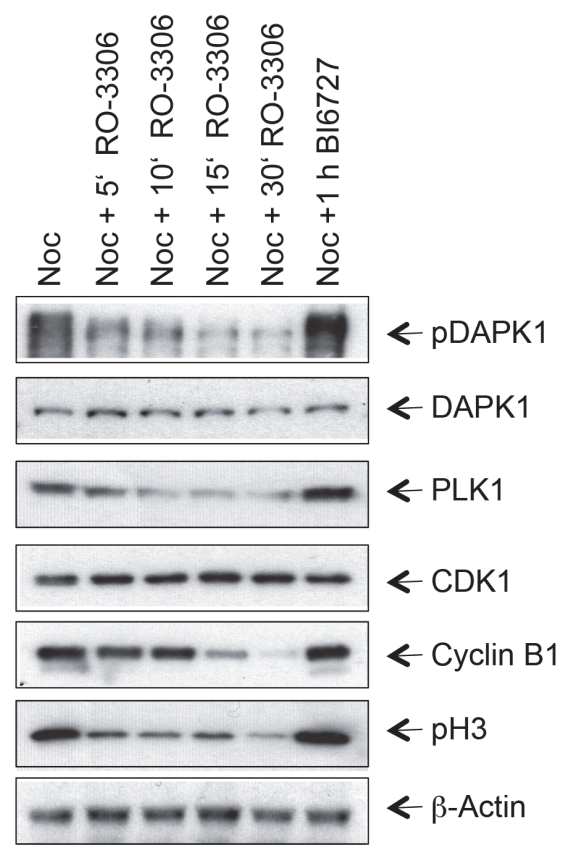

Abstract 1003 Figure 1 